\title{
Staphylococcus aureus DsbA is a membrane-bound lipoprotein with thiol-disulfide oxidoreductase activity
}

\author{
Journal Article \\ Author(s): \\ Dumoulin, Alexis; Grauschopf, Ulla; Bischoff, Markus; Thöny-Meyer, Linda; Berger-Bächi, Brigitte \\ Publication date: \\ 2005-09 \\ Permanent link: \\ https://doi.org/10.3929/ethz-b-000031190 \\ Rights / license: \\ In Copyright - Non-Commercial Use Permitted \\ Originally published in: \\ Archives of Microbiology 184(2), https://doi.org/10.1007/s00203-005-0024-1
}




\section{Alexis Dumoulin · Ulla Grauschopf · Markus Bischoff Linda Thöny-Meyer · Brigitte Berger-Bächi \\ Staphylococcus aureus DsbA is a membrane-bound lipoprotein with thiol-disulfide oxidoreductase activity}

Received: 4 May 2005/ Revised: 8 June 2005/ Accepted: 22 June 2005/Published online: 22 September 2005

(C) Springer-Verlag 2005

\begin{abstract}
DsbA proteins, the primary catalysts of protein disulfide bond formation, are known to affect virulence and penicillin resistance in Gram-negative bacteria. We identified a putative DsbA homologue in the Grampositive pathogen Staphylococcus aureus that was able to restore the motility phenotype of an Escherichia coli $d s b A$ mutant and thus demonstrated a functional thiol oxidoreductase activity. The staphylococcal DsbA (SaDsbA) had a strong oxidative redox potential of $-131 \mathrm{mV}$. The persistence of the protein throughout the growth cycle despite its predominant transcription during exponential growth phase suggested a rather long half-life for the SaDsbA. SaDsbA was found to be a membrane localised lipoprotein, supporting a role in disulfide bond formation. But so far, neither in vitro nor in vivo phenotype could be identified in a staphylococcal $d s b A$ mutant, leaving its physiological role unknown. The inability of SaDsbA to interact with the E. coli DsbB and the lack of an apparent staphylococcal DsbB homologue suggest an alternative re-oxidation pathway for the SaDsbA.
\end{abstract}

Keywords DsbA Staphylococcus aureus . Disulfide $\cdot$ Lipoprotein $\cdot$ Cysteine

\footnotetext{
A. Dumoulin · M. Bischoff · B. Berger-Bächi

Department of Medical Microbiology, University of Zürich, Gloriastr 32, 8006 Zürich, Switzerland

E-mail: bberger@immv.unizh.ch

Tel.: + 41-44-6342650

Fax: +41-44-6344906

L. Thöny-Meyer

Institute of Microbiology, ETH Hönggerberg,

Wolfgang-Pauli-Str 10, 8093 Zürich, Switzerland

U. Grauschopf

Institute of Molecular Biology, ETH Hönggerberg,

Schafmattstr 20, 8093 Zürich, Switzerland
}

\section{Introduction}

Disulfide bonds between cysteine residues support the correct folding of proteins and maintain their structural integrity and activity. Disulfide bond containing proteins are usually localised in extracytoplasmic compartments or secreted into the medium, but are rare in the reducing environment of the cytoplasm. Although disulfide formation can occur spontaneously under oxidising conditions, this reaction is unspecific and slow (Anfinsen 1973). In vivo, enzymatic systems ensure efficient and correct disulfide bond formation. The primary catalyst of this reaction in Escherichia coli is the extensively studied periplasmic thiol-disulfide oxidoreductase DsbA. It is a monomeric $21-\mathrm{kDa}$ protein containing a redox active C-X-X-C sequence embedded in a thioredoxin fold, typical for the proteins of this family (reviewed in Kadokura et al. 2003). Upon catalysing disulfide bond formation, the two cysteines of DsbA become reduced and are subsequently re-oxidised by the inner membrane protein DsbB, which passes the electrons to the electron transfer chain via quinone reduction (Guilhot et al. 1995; Kishigami et al. 1995; Kishigami and Ito 1996; Bader et al. 1998, 1999).

Escherichia coli $d s b A$ mutants have a pleiotropic phenotype and, amongst others, lack motility due to the improper assembly of the flagellar motor (Jones et al. 1990; Dailey and Berg 1993). Interestingly, $d s b A$ mutants of several Gram-negative pathogenic bacteria also exhibit a reduced virulence, due to the absence of critical disulfide bonds in exotoxins, adherence factors or pili (Yu and Kroll 1999; Yu et al. 2001; Stenson and Weiss 2002; Ha et al. 2003; Burall et al. 2004; Miki et al. 2004). So far, an involvement of a DsbA homologue in virulence of a Gram-positive pathogen has not been documented.

In Gram-negative bacteria, disulfide bond formation occurs in the periplasm. Gram-positive bacteria do not possess a classic periplasmic compartment, and very little is known about disulfide bond formation in these organisms. The Bacillus brevis Bdb was the first DsbA 
Table 1 Bacterial strains and plasmids
Relevant properties

Source or reference

\begin{tabular}{|c|c|c|}
\hline & Relevant properties & Source or reference \\
\hline \multicolumn{3}{|l|}{ Strains } \\
\hline \multicolumn{3}{|l|}{ Escherichia coli } \\
\hline Rosetta DE3 & BL21 derivative & Novagen \\
\hline AD13 & JCB571, pAD2 & This study \\
\hline AD 15 & JCB571, pBR322 & This study \\
\hline AD28 & JCB571, pAD11 & This study \\
\hline AD29 & JCB571, pISC2 & This study \\
\hline AD44 & Rosetta DE3, pmSaDsbA, Ap ${ }^{\mathrm{r}}$ & \\
\hline AD59 & $\mathrm{JCB} 818, \mathrm{pAD} 2, \mathrm{Km}^{\mathrm{r}}, \mathrm{Ap}^{\mathrm{r}}$ & This study \\
\hline AD60 & $\mathrm{JCB} 818, \mathrm{pAD} 11, \mathrm{Km}^{\mathrm{r}}, \mathrm{Ap}^{\mathrm{r}}$ & This study \\
\hline AD189 & $\mathrm{JCB} 818, \mathrm{pBR} 322, \mathrm{Km}^{\mathrm{r}}, \mathrm{Ap}^{\mathrm{r}}$ & This study \\
\hline AD 190 & $\mathrm{JCB} 818, \mathrm{pEC} 52, \mathrm{Km}^{\mathrm{r}}, \mathrm{Ap}^{\mathrm{r}}$ & This study \\
\hline AD191 & $\mathrm{JCB} 570, \mathrm{pBR} 322, \mathrm{Km}^{\mathrm{r}}, \mathrm{Ap}^{\mathrm{r}}$ & This study \\
\hline JCB570 & MC1000 phoR zih12::Tn 10 & Bardwell et al. 1991 \\
\hline JCB571 & JCB570 dsb A::kanl & Bardwell et al. 1991 \\
\hline JCB 818 & $\mathrm{MC} 1000 d s b A: * k a n 1, d s b B: * k a n, \mathrm{Km}^{\mathrm{r}}$ & Jonda et al. 1999 \\
\hline \multicolumn{3}{|c|}{ Staphylococcus aureus } \\
\hline $8325-4$ & NCTC8325 derivative, cured of all prophages & Peng et al. 1988 \\
\hline AD19 & RN4220 dsbA::tet; $\mathrm{Tc}^{\mathrm{r}}$ & This study \\
\hline AD21 & $\mathrm{BB} 270$ dsb A $\because$ tet $L ; \mathrm{Tc}^{\mathrm{r}}$, methicillin resistant $\left(\mathrm{Mc}^{\mathrm{r}}\right)$ & This study \\
\hline AD22 & Newman dsbA::tetL; $\mathrm{Tc}^{\mathrm{r}}$ & This study \\
\hline AD23 & MSSA1112 dsbA::tetL; $\mathrm{Tc}^{\mathrm{r}}$ & This study \\
\hline AD 188 & $8325-4$ dsb A : ${\text { tet } L ; \mathrm{Tc}^{\mathrm{r}}}^{\mathrm{r}}$ & This study \\
\hline BB270 & NCTC8325 mec; $\mathrm{Mc}^{\mathrm{r}}$ & $\begin{array}{l}\text { Berger-Bächi and } \\
\text { Kohler } 1983\end{array}$ \\
\hline MB261 & $\mathrm{BB} 270(d s b A p: \because l u c+)-\mathrm{pBT}$ & This study \\
\hline Newman & ATCC 25904, clinical isolate & $\begin{array}{l}\text { Duthie and } \\
\text { Lorenz } 1952\end{array}$ \\
\hline RN4220 & $8325-4$; restriction negative & $\begin{array}{l}\text { Rhoads Kraemer } \\
\text { and Iandolo } 1990\end{array}$ \\
\hline \multicolumn{3}{|l|}{ Plasmids } \\
\hline pAD2 & $\begin{array}{l}\text { pBR322 derivative; containing a } 1.4 \mathrm{~kb} \\
\text { fragment of } S \text {. aureus } \mathrm{BB} 270 \text { encoding SA2 } 198 \\
\text { and } d s b A ; \mathrm{Tc}^{\mathrm{r}}, \mathrm{Ap}^{\mathrm{r}}\end{array}$ & This study \\
\hline pAD7 & $\begin{array}{l}\text { pEC4 derivative ; containing the double crossover } \\
\text { construct for the inactivation of } d s b A \text { in } S \text {. aureus; } \\
\mathrm{Tc}^{\mathrm{r}} ; \mathrm{Em}^{\mathrm{r}} ; \mathrm{Ap}^{\mathrm{r}}\end{array}$ & \\
\hline pAD11 & $\begin{array}{l}\text { pISC2 derivative; containing the sequence for a } \\
\text { translational fusion of the OmpA signal peptide } \\
\text { and the truncated (aa 19-199) DsbA of } S \text {. aureus } \\
\text { under the control of an arabinose inducible } \\
\text { promoter; Ap }\end{array}$ & This study \\
\hline pBR322 & E. coli cloning vector; $\mathrm{Tc}^{\mathrm{r}}, \mathrm{Ap}^{\mathrm{r}}$ & Bolivar et al. 1977 \\
\hline pBT & $\begin{array}{l}\text { S. aureus suicide vector; containing the tet } L \text { gene; } \\
\mathrm{Tc}^{\mathrm{r}}\end{array}$ & Giachino et al. 2001 \\
\hline $\mathrm{pBT} d s b A: \because l u c+$ & $\begin{array}{l}\text { pBT with } 1 \mathrm{~kb} \text { PCR fragment of the } d s b A \\
\text { promoter from strain BB270 fused to the } \\
\text { luciferase gene } l u c+; \mathrm{Tc}^{\mathrm{r}}\end{array}$ & This study \\
\hline pEC4 & Suicide vector ; containing the tet $L$ gene; $\mathrm{Tc}^{\mathrm{r}}$ & Brückner 1997 \\
\hline pEC52 & $\begin{array}{l}\text { pISC2 derivative; containing the sequence for a } \\
\text { translational fusion of the OmpA signal peptide } \\
\text { and the truncated CcmG of } E \text {. coli under the } \\
\text { control of an arabinose inducible promoter; } \mathrm{Ap}^{\mathrm{r}}\end{array}$ & Fabianek et al. 1998 \\
\hline pET11a & E. coli cloning vector; $\mathrm{Ap}^{\mathrm{r}}$ & Novagen \\
\hline pISC2 & E. coli expression vector; $A p^{r}$ & Thöny-Meyer et al. 1998 \\
\hline pmSaDsbA & $\begin{array}{l}\text { pET11a derivative; encoding a truncated DsbA } \\
\text { without signal sequence; } \mathrm{Ap}^{\mathrm{r}}\end{array}$ & This study \\
\hline pSP- $l u c+$ & Firefly luciferase cassette vector; $\mathrm{Ap}^{\mathrm{r}}$ & Promega \\
\hline pSP- $d s b A: \because l u c+$ & $\begin{array}{l}1 \mathrm{~kb} \text { PCR fragment of } d s b A \text { promoter from strain } \\
\mathrm{BB} 270 \text { in pSP- } l u c+; \mathrm{Ap}^{\mathrm{r}}\end{array}$ & This study \\
\hline
\end{tabular}

homologue of a Gram-positive bacterium shown to have thiol oxidase activity by complementing the motility of an $E$. coli $d s b A$ mutant (Ishihara et al. 1995). Subsequently, the Bacillus subtilis $\mathrm{BdbC}$ and $\mathrm{BdbD}$ were demonstrated to be functional homologues of the $E$. coli DsbA and DsbB, respectively, and to be involved in the development of competence (Erlendsson and Hederstedt 2002; Meima et al. 2002). Although some of the secreted virulence factors produced by the human pathogen Staphylococcus aureus, such as all staphylococcal enterotoxins and the beta-haemolysin, are known to contain disulfide bonds in their active forms (Hovde et al. 
Table 2 Primers

\begin{tabular}{llll}
\hline Primers & Sequences $5^{\prime}-3^{\prime 1}$ & $\begin{array}{l}\text { Position of } \\
\text { the } 3^{\prime} \text { end }^{2}\end{array}$ & $\begin{array}{l}\text { Restriction } \\
\text { site }\end{array}$ \\
\hline Seq1 & catgatgcggattatcatg & -38 & \\
Seq2 & ctgcatgactcgcacgcgaacc & 253 & \\
Seq3 & ctcgtacaattaaaattgtc & 33 & \\
Seq4 & gctataatagttgttgtact & -71 & \\
Pr1f & gggaattcatgtttgacggtgtatag & -431 & EcoRI \\
Pr1r & ggaagcttacttttgtcataagtgcc & 914 & HindIII \\
PEX1 & taatgttagtaatttttagtc & 3 & \\
PEX2 & atcaccgttgcctttttgg & -256 & \\
PEX3 & cacaaatcgcttcaatgctc & -385 & Asp718 \\
DsbApf & gaggtaccaattcaccgtctgcattgcg & -977 & KpnI \\
DsbApr & tagccatggtaactcctgttcatatctagttcg & -26 & NheI \\
SaDsbAf & cctagctagcatgcatcaccatcaccaccatggtaaaaaagaatcagcaacgacatcc & 84 & Bam HI \\
SaDsbAr & ccgcggatcctatttatttatctttaataacttctcataactttc & 525 & StuI \\
Pr3f & cagaggcctcaaaaaaagaatcagcaacg & 78 & EcoRI \\
Pr3r & cgggaattcgaagaatcaatacctatcctg & 755 & \\
Pr4f & cgaaagtgccacctgacg & & \\
Pr4r & aactaatggtttgccgttttc & 88 & \\
TetL + & ccatattgttgtataagtg & & \\
TetL - & cctgttataaaaaaaggatc & & \\
\hline
\end{tabular}

1994; Dziewanowska et al. 1996), the mechanism by which these disulfide bonds are formed has never been examined. In this study we describe the identification and functional characterisation of a putative DsbA homologue (SA2197) in $S$. aureus and analyse its importance for virulence and antibiotic resistance.

\section{Materials and Methods}

Bacterial strains, plasmids, and media

The strains and plasmids used in this study are listed in Table 1 . The cells were grown at $37^{\circ} \mathrm{C}$ in Luria-Bertani (LB) medium. When required, tetracycline $10 \mu \mathrm{g} / \mathrm{ml}$, or ampicillin $100 \mu \mathrm{g} / \mathrm{ml}$ were added.

DNA manipulations

Preparation of chromosomal and plasmid DNA, PCR, and Southern blot analysis were performed by standard procedures (Sambrook et al. 1989). Probes were labelled with the PCR DIG Probe Synthesis Kit (Roche, Rotkreuz, CH, USA), following the manufacturer's instructions. Transductions were done with phage $80 \alpha$ as described previously (Berger-Bächi 1983). Primers used for PCR, sequencing and primer extension reactions are listed in Table 2.

\section{Sequence analysis}

Direct chromosomal sequencing of the SA2198- $d s b A$ operon of strain BB270 was done according to the protocol of A. Wada (Wada 2001) on a ABI Prism 310 Sequencer (Perkin Elmer) with the primers Seq1, Seq2,
Seq3, Seq4 and PEX2. The sequences were compared to genome sequences of $S$. aureus NCTC8325 (NC_002954), Mu50 (NC_002758), N315 (NC_002745), MW2 (NC_003923) (Kuroda et al. 2001), COL (TIGR), MSSA476 (NC_002953) and MRSA252 (NC_002952). Homology searches were performed using the program BLAST (http://www.ncbi.nlm.nih.gov/BLAST) (Altschul et al. 1990). The sequence was deposited in GenBank under the accession number AF321274.

\section{Isolation and analysis of total RNA}

Total RNA was isolated according to the protocol of Cheung (Cheung et al. 1994), using the FastRNA kit (BIO 101, Vista, CA, USA). Northern blot analysis was performed as described by Sambrook (Sambrook et al. 1989). The transcriptional start was determined by primer extension using the ${ }^{32}$ P-labeled primers PEX1, PEX2 and PEX3 as described by Rossi (2003).

\section{$d s b A$ promoter-reporter gene construct}

A $1 \mathrm{~kb}$ DNA fragment covering the SA2198-dsb $A$ promoter region $(d s b A p)$ of $S$. aureus was amplified using the primers DsbApf and DsbApr, digested with KpnI and $N c o I$ and cloned in front of the luciferase gene of plasmid pSP- $l u c+$. A $2.6 \mathrm{~kb} K p n \mathrm{I}-E c o \mathrm{RI}$ fragment, including the $d s b A$ promoter region fused to the luciferase coding region was subsequently subcloned into suicide plasmid pBT (Giachino et al. 2001) yielding plasmid pBT $d s b A p: \because l u c+$, which was electroporated into RN4220. A co-integrate was selected and used as a donor to transduce strain BB270, resulting in strain MB261. The luciferase activity was measured as described earlier (Bischoff et al. 2001). 
SaDsbA-isolation and antibody preparation

The primers SaDsbAf and SaDsbAr were used to amplify a fragment encoding the presumed mature part of SaDsbA lacking its signal sequence ( $\mathrm{mSaDsbA}$ ). The fragment was cloned into pET11a using NheI and BamHI restriction sites. The cloning procedure did add four supplementary amino acids M-A-S-M to the N-terminus of $\mathrm{mSaDsbA}$, thereby introducing a new start methionine to the $\mathrm{mSaDsbA}$. The resulting plasmid pmSaDsbA was transformed into Rosetta DE3 cells (Novagen) for overexpression. Cells were grown in LB medium to an $\mathrm{OD}_{600}$ of 0.8 and induced with $1 \mathrm{mM}$ IPTG. After $3 \mathrm{~h}$, cells were collected by centrifugation and resuspended in $20 \mathrm{mM}$ Tris $/ \mathrm{HCl} \mathrm{pH}$ 8.0. The cells were disrupted (Cell Cracker, EMBL, Heidelberg Germany), and the debris separated by centrifugation for $30 \mathrm{~min}$ at $15,000 \mathrm{~g}$. The supernatant was applied onto a QA-52 anion-exchange column (Whatman, Brentfort, UK) pre-equilibrated with $20 \mathrm{mM}$ Tris $/ \mathrm{HCl} \mathrm{pH} \mathrm{8.0.} \mathrm{Proteins} \mathrm{were} \mathrm{eluted} \mathrm{with} \mathrm{a} 0$ $1 \mathrm{M}$ gradient of $\mathrm{NaCl}$ in $20 \mathrm{mM}$ Tris/ $\mathrm{HCl} \mathrm{pH}$ 8.0. Fractions containing $\mathrm{mSaDsbA}$ were pooled, dialysed against $20 \mathrm{mM}$ HEPES/NaOH pH 8.0 and applied onto a SE52 cation-exchange column (Whatman). mSaDsbA was eluted with a gradient of $0-1 \mathrm{M} \mathrm{NaCl}$ in $20 \mathrm{mM}$ HEPES/ $\mathrm{NaOH}$ pH 8.0. One milligram of the eluted, homogenous $\mathrm{mSaDsbA}$ was used to raise chicken antibodies (DAVIDS Biotechnologie, Regensburg, Germany).

Fluorescence spectroscopy and determination of the redox potential

The redox potential of mSaDsbA was determined essentially as previously described (Grauschopf et al. 2003) by measuring the redox equilibrium of $\mathrm{mSaDsbA}$ with glutathione using a specific fluorescence intensity change at $327 \mathrm{~nm}$ between the oxidised and reduced state of the protein.

\section{Localisation of SaDsbA}

Exponentially growing Newman cells harvested at $\mathrm{OD}_{600} 2$ were disrupted and fractionated into supernatant, cell wall, cell membrane and cytoplasm, according to Schneewind (1993). Protein fractions corresponding to a $1-\mathrm{ml}$ culture were subjected to Western analysis using antigen-purified anti-DsbA antibodies at a dilution of 1:5,000. To demonstrate the lipoprotein nature of SaDsbA, growing cells were challenged with $50 \mu \mathrm{g} / \mathrm{ml}$ globomycin (Sankyo Corp., Tokyo, Japan) at $\mathrm{OD}_{600} 0.4$ and harvested at $\mathrm{OD}_{600} 2$. Aliquots containing $15 \mu \mathrm{g}$ whole protein were subjected to Western blot analysis using the conditions given above.

Complementation of an E. coli $d s b A$ mutant by the $d s b A$ gene of $S$. aureus

A 1.4-kb fragment covering the SA2198- $d s b A$ operon as well as the SA2198- $d s b A$ promoter was amplified using the primers Pr1f and Pr1r. The obtained insert was cloned into the EcoRV site of pBR322, yielding pAD2. Plasmid pAD11, carrying a translational in-frame fusion of the E. coli OmpA signal peptide, MKKTAIAIAVALAGFATVAQA, with the putative mature part of SaDsbA (aa 19-199) was constructed by amplifying a 735-bp fragment encoding an SaDsbA lacking its $18 \mathrm{~N}$ terminal amino acids with the primers $\operatorname{Pr} 3 f$ and $\operatorname{Pr} 3 r$, and cloning it into the StuI-EcoRI restricted pEC52 (Fabianek et al. 1998). In this construct the codons for cysteine 19 and glycine 20 were changed to codons for alanine and serine, respectively, thereby eliminating the signal peptidase II recognition site of SaDsbA.

To determine the complementation of the non-motile E. coli $d s b A$ mutant strain JBC571 (Bardwell et al. 1991), plasmids pAD2, pAD11 as well as the empty vectors pBR322 and pISC2 were electroporated into $E$. coli JCB571. The motility of the resulting strains AD13, $\mathrm{AD} 28, \mathrm{AD} 15$ and AD29, respectively, was tested by placing filter discs of $6 \mathrm{~mm}$ diameter (bioMérieux) soaked in the respective overnight cultures, onto an LB plate containing $0.3 \%$ agar and $0.2 \%$ arabinose and incubating for $12.5 \mathrm{~h}$ at $37^{\circ} \mathrm{C}$. To test the dependence of the complementation on the presence of DsbB, plasmids pAD2, pAD11, pBR322 and pISC2 were electroporated into the $E$. coli $d s b A d s b B$ double mutant JCB818, yielding strains AD59, AD60, AD189 and AD190, respectively. As a control, pBR322 was electroporated into the wild-type strain JCB570, yielding AD191. The dependence of the complementation of the $d s b A$ and $d s b A d s b B$ mutants on the presence of cystine was demonstrated by growing the strains overnight in liquid M9 medium containing $4 \mu \mathrm{g} / \mathrm{ml}$ of each amino acids except cysteine, $0.4 \%$ glycerol and $100 \mu \mathrm{g} / \mathrm{ml}$ ampicillin. Thirty microlitre of the overnight culture, resuspended to an $\mathrm{OD}_{600}$ of 0.2 , were placed on $6 \mathrm{~mm}$ filter discs which were transferred to an M9 agar plate containing $0.3 \%$ agar, $0.2 \%$ arabinose and $4 \mu \mathrm{g} / \mathrm{ml}$ of each amino acid except cysteine. When required, $25 \mu \mathrm{g} / \mathrm{ml}$ cystine was added to the medium. The distance reached by motile swarming cells was determined after overnight incubation.

\section{Insertional inactivation of $d s b A$}

The $d s b A$ gene from $S$. aureus was inactivated by replacing a $249 \mathrm{bp}$ internal fragment with a $1.6-\mathrm{kb}$ tet $L$ cassette. An 845-bp fragment upstream of $d s b A$ was amplified using pAD2 as a template and the primers $\operatorname{Pr} 4 \mathrm{f}$ and Pr4r, digested with EcoRI, and inserted into the 4.7$\mathrm{kb}$ fragment EcoRI-HpaI fragment of pAD2. The three bases GTT at the 3'-end of the insert restored an $\mathrm{HpaI}$ site after ligation with the vector. The $1.6-\mathrm{kb}$ tet $L$ fragment was amplified by PCR from plasmid pBT using the primers TetL + and TetL- and inserted into the restored $\mathrm{HpaI}$ site. From this product the 2.7-kb Eco RI-BamHI fragment was excised and inserted into the corresponding sites of $\mathrm{pEC} 4$, yielding plasmid pAD7. Tetracycline 
resistant clones obtained after electroporation of pAD7 into the S. aureus RN4220 were screened for loss of erythromycin resistance. About $5 \%$ of the tetracycline resistant clones were sensitive to erythromycin, indicating loss of the plasmid backbone due to a double crossover. The $\operatorname{dsbA::tet} L$ allele of the resulting strain AD19 was transferred to strains BB270, Newman, MSSA112, and $8325-4$ by phage transduction, resulting in strains $\mathrm{AD} 21, \mathrm{AD} 22, \mathrm{AD} 23$, and $\mathrm{AD} 188$ respectively. The deletion of the $d s b A$ fragment on the chromosome was confirmed by Southern blot analysis.

\section{Characterisation of the $S$. aureus $d s b A$ mutant}

Susceptibility to dithiothreitol (DTT) was tested by placing sterile filter discs of $6 \mathrm{~mm}$ diameter impregnated with $20 \mu \mathrm{l}$ of a $1 \mathrm{M}$ DTT on a lawn of cells diluted to McFarland 0.5 in $0.8 \% \mathrm{NaCl}$ and spread on MüllerHinton plates. The inhibition zone was measured after overnight incubation at $37{ }^{\circ} \mathrm{C}$. Susceptibility to antibiotics was assessed by E-test (AB Biodisk, Solna, Sweden) following the recommendations of the manufacturer. The relative resistance to oxacillin of the strain pair BB270/AD21 was compared on plates containing a gradient of oxacillin from 0 to $200 \mu \mathrm{g} / \mathrm{ml}$.

\section{Measurement of the haemolytic activity}

The haemolytic activities of strain 8325-4 and its $d s b A$ defective derivative AD188 were essentially determined following the protocol of Nilsson and co-workers (Nilsson et al. 1997). After incubation of the washed erythrocytes with diluted culture supernatant, the samples were centrifuged for $1 \mathrm{~min}$ at $7000 \mathrm{~g}$. The optical density at $415 \mathrm{~nm}$ due to the released haemoglobin was measured and plotted against the dilution factor of the culture supernatant.

\section{Animal models}

Strain Newman and its $d s b A$ mutant AD22 were used in two pathogenicity assays. The Caenorhabditis elegans killing assay was performed according to the protocol by Sifri and co-workers (Sifri et al. 2003). In the mouse model for septic arthritis, the protocol described by Bremell and co-workers (Bremell et al. 1991) was followed, using 15 mice and a bacterial load of $6 \times 10^{6}$ cells.

\section{Results}

Identification of a putative DsbA homologue

B. subtilis $\mathrm{BdbD}$ was previously identified as a close E. coli DsbA homologue in a Gram-positive organism
(Fabianek et al. 2000). A BLAST search for Bdb homologues in the $S$. aureus NCTC8325 genome database resulted in the identification of a 199-amino acid ORF with $41 \%$ similarity to BdbD that was tentatively termed SaDsbA. No further Dsb or Bdb homologues were found in any of the published genome sequences of $S$. aureus. Direct chromosomal sequencing of the NCTC8325 derivative BB270 over the region covering $d s b A$ showed an exact match with the corresponding published genome sequences of NCTC8325 and COL. In S. aureus MW2, Mu50, N315, MRSA252 and MSSA476, a G to A transition causes the valine residue at position 38 to be changed to a isoleucine. In addition, a $G$ to $T$ transversion causes the change of leucine at position 52 to a phenylalanine in strain MRSA252.

The deduced sequence of the $20 \mathrm{~N}$-terminal amino acids of the $S$. aureus DsbA revealed the characteristics of a lipoprotein signal sequence with an ILTACG predicted signal peptidase II cleavage site (PROSITE motive PS00013) (Tjalsma et al. 2000; Sutcliffe and Harrington 2002), suggesting SaDsbA to be an extracytoplasmic lipoprotein with a lipid attachment site at cysteine 19.

The predicted size of 181 aa of the deduced mature SaDsbA was similar to that of DsbA from other bacteria, and conserved residues were found over the entire length of its primary structure (Fig. 1). SaDsbA harbours, with its C-P-Y-C motif, the C-X-X-C active site characteristic for thiol-disulfide oxidoreductases, and possesses a proline residue at position 154, which aligns well with the $c i s$-proline at position 151 of $E$. coli DsbA (Fig. 1). The cis-proline, strongly conserved in all members of the thioredoxin family, and positioned close to the C-X-X-C motif in their native form (Martin 1995), has a critical role in the resolution of the mixed disulfide intermediate (Kadokura et al. 2004) and affects the stability of DsbA proteins (Charbonnier et al. 1999).

Upstream of the $S$. aureus $d s b A$, and separated by 21 nucleotides, a 360-bp open reading frame (ORF), SA2198, was identified. This ORF encodes a putative protein of 120 amino acids that shares no apparent similarity to any other protein with known function. SA2198 appears to have a lipoprotein signal sequence with a FISGCG predicted signal peptidase II cleavage site. The sequence of this ORF was found to be identical in all the published $S$. aureus genome sequences, suggesting that its gene product is functional in $S$. aureus. Additionally, a similar genetic organisation was found in the closely related Staphylococcus epidermidis, with the two predicted lipoproteins, SE1987 sharing 68\% sequence similarity with SaDsbA, and SE1988 sharing $65 \%$ similarity with SA2198.

Analysis of the $d s b A$ transcript

Northern blot analyses with probes covering either $d s b A$ or SA2198- $d s b A$, yielded a single $1.3-\mathrm{kb}$ transcript 

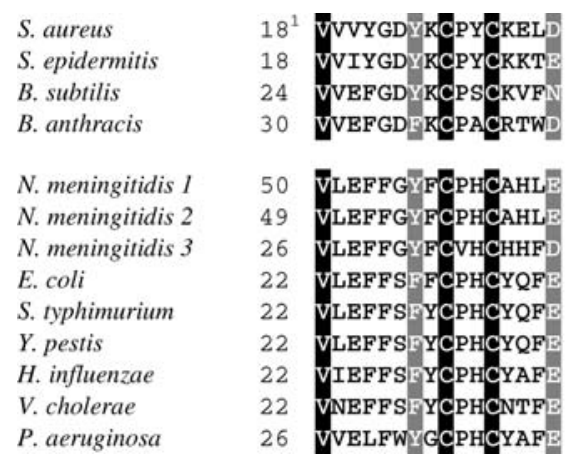

Fig. 1 Alignment of the conserved active site and of the cis-proline region of some members of the DsbA protein family. The alignment was performed with the program ClustalW (Thompson et al. 1994) using the default parameters. Identical residues are underlayed in black. Dark grey and grey shaded residues correspond to conserved and semi-conserved substitutions, respectively. ${ }^{1}$ The position indicated refers to the first aa in the alignment of the mature protein. The position of the cleavage site was predicted by the program SignalP using the standard parameters (Bendtsen et al. 2004). ${ }^{2}$ The indicated length refers to the mature protein. Sequences shown are from $S$. aureus, S. epidermidis, B. subtilis, Bacillus anthracis, Neisseria meningitidis, E. coli, Salmonella typhimurium, Yersinia pestis, Haemophilus influenzae, Vibrio cholerae and Pseudomonas aeruginosa

(Fig. 2a), indicating that SA2198 and $d s b A$ are cotranscribed, thereby forming a bi-cistronic operon. The level of the $1.3-\mathrm{kb}$ transcript was high during early exponential growth (30 $\mathrm{min}$ to $2 \mathrm{~h}$ ) and strongly decreased thereafter $(3$ and $5 \mathrm{~h}$ ). A $d s b A$ promoter-luciferase reporter gene fusion, co-integrated into the $S$. aureus chromosome of strain MB261, confirmed the growth phase-dependent expression pattern of SA2198$d s b A$ (Fig. 2b). Western blot analysis, however, demonstrated SaDsbA to be present at all time points analysed, irrespective of the transcription pattern of its gene (Fig. 2c).

Primer extension experiments, using primers binding either within $d s b A$ or SA2198 coding sequences, yielded one transcriptional start point 42 bp upstream of SA2198 (Fig. 3), confirming the co-expression of these two genes. The identified transcriptional start point is preceded by a sequence (TTGTAG-20-TATAGT), which is fairly well resembling the consensus promoter sequence of the house-keeping $\sigma^{\mathrm{A}}$ factor (TTGACA-16-18-TATAAT) (Helmann 1995; Rao et al. 1995), with the predicted -35 and -10 regions being separated, however, by an unusually long spacer region of 20 nucleotides.

Production of SaDsbA in E. coli and determination of its redox potential

We produced the predicted mature part of SaDsbA in the cytoplasm of E. coli strain AD44 containing plasmid pmSaDsbA. After the two-step purification using an anion-exchange and a cation-exchange column, $80 \mathrm{mg}$ of pure $\mathrm{mSaDsbA}$ were obtained from an original 61

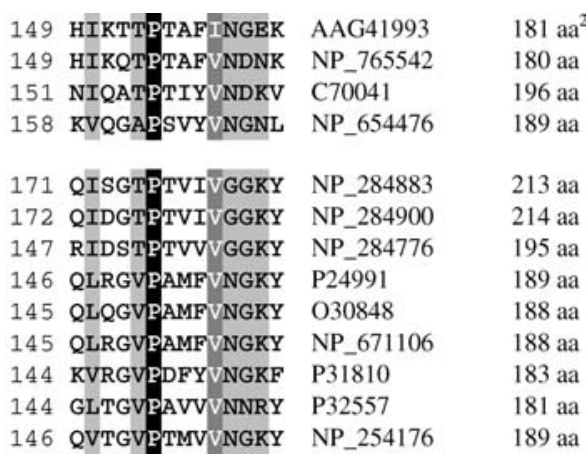

culture. We found that oxidised mSaDsbA, similarly to its E. coli homologue exhibited an increase in tryptophane fluorescence at $327 \mathrm{~nm}$ upon reduction of the disulfide bond by dithiothreitol. We made use of this redox-state-dependent fluorescence to determine the intrinsic redox potential of mSaDsbA. By equilibrating the protein with different glutathione redox buffers, a
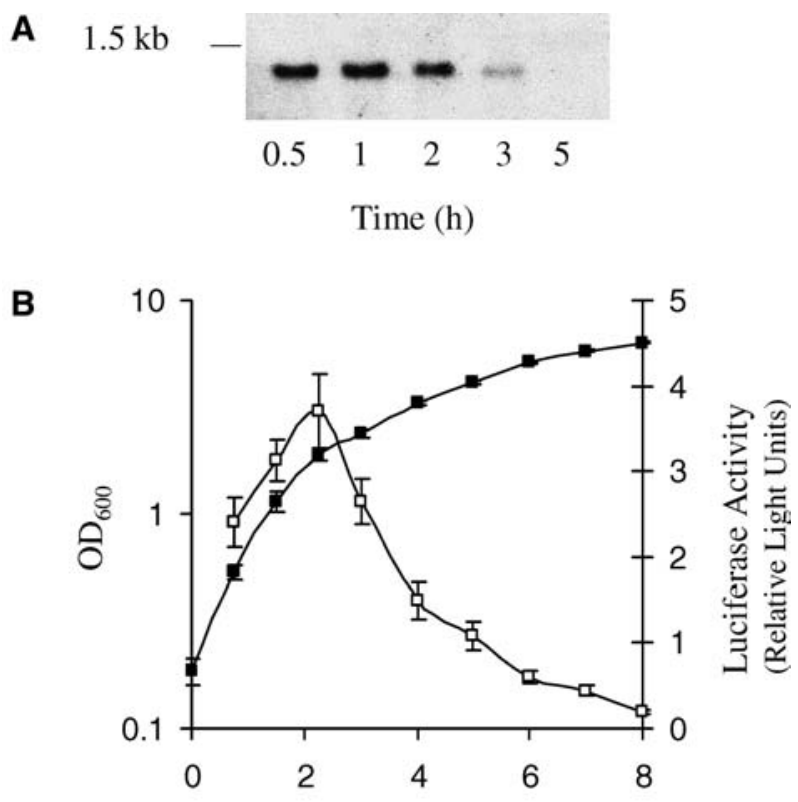

Time (h)

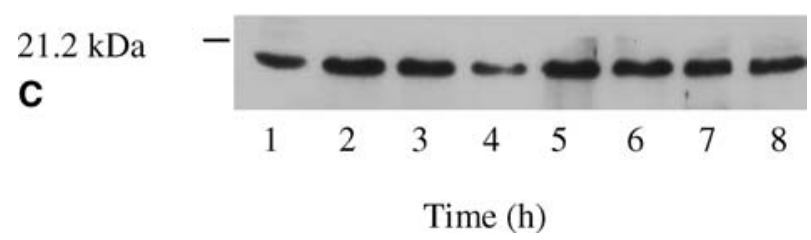

Fig. 2 Expression pattern of $d s b A$ transcript during growth. A Northern blot analyses of $d s b A$ expression in strain BB270 using a DIG-labelled DNA-probe specific for SA2198 and $d s b A$. B The transcriptional activity of the $d s b A$ promoter was determined by measuring the luciferase activity of a $d s b A: \because l u c+$ fusion (open symbols). Bacterial growth was measured as the $\mathrm{OD}_{600}$ (solid symbols). C Detection of SaDsbA by Western blot analysis. Fifteen micrograms obtained from whole-cell extracts were loaded in each lane 

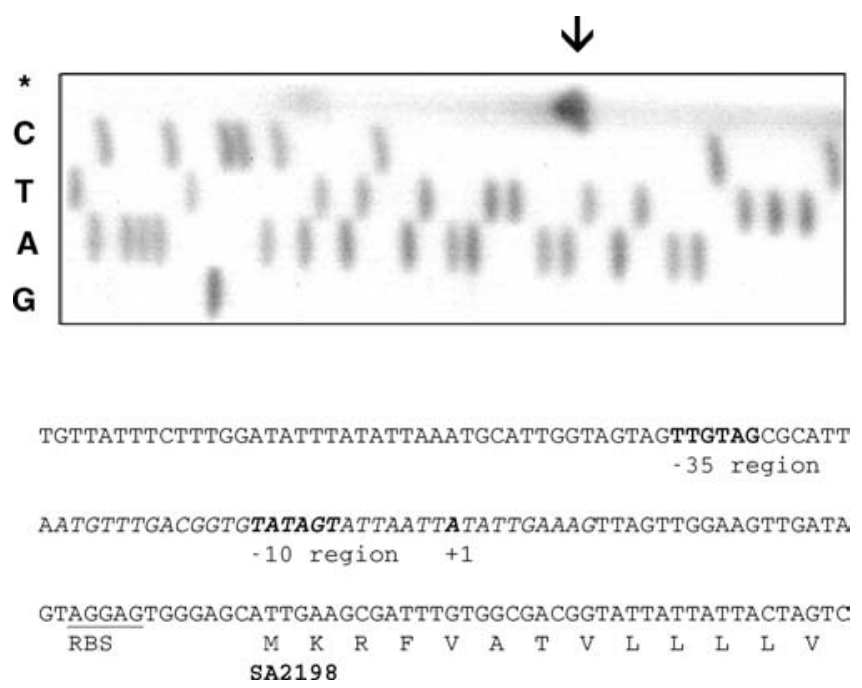

Fig. 3 Determination of the transcription start site of the SA2198 $d s b A$ transcript by primer extension. The signal detected using the $\gamma-{ }^{32} \mathrm{P}$ labelled primer PEX3 is designated by an arrow. The same start site was obtained with the primers PEX1 and PEX2 (data not shown). The portion of the sequence represented in the picture is written in italics. The -10 region, the mapped transcriptional start, the ribosome binding site and the first amino acids of SA2198 are indicated

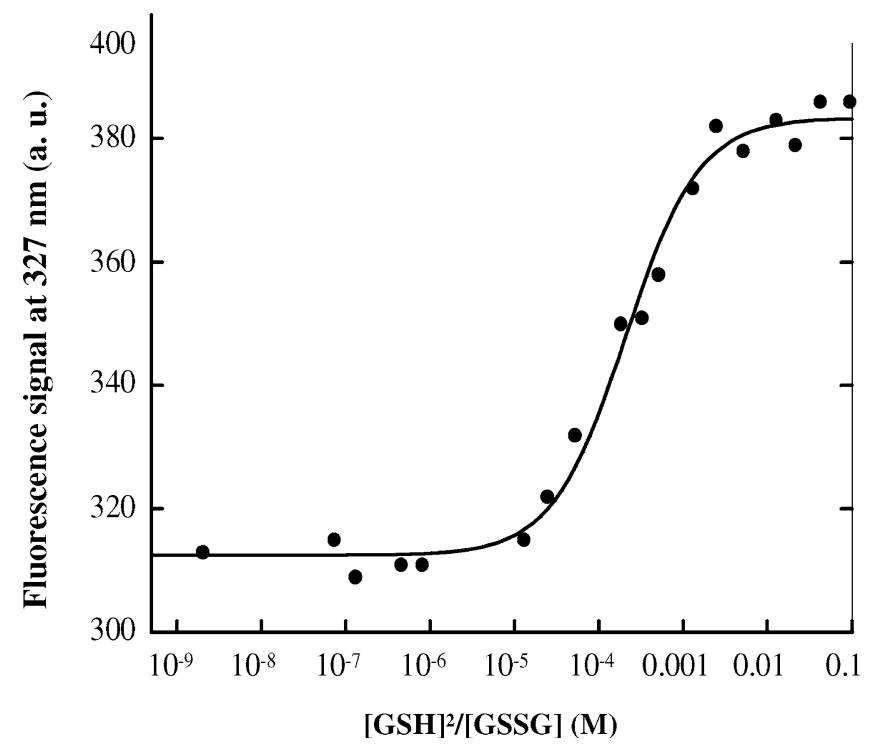

Fig. 4 Redox equilibrium of SaDsbA with glutathione. SaDsbA was incubated in the presence of $1 \mathrm{mM}$ GSSG and varying concentrations of GSH in $100 \mathrm{mM}$ sodium phosphate, $\mathrm{pH} 7.0$ at $25^{\circ} \mathrm{C}$. The tryptophane fluorescence at $327 \mathrm{~nm}$ was recorded, and data were fitted as described (Grauschopf et al. 2003)

single transition with an equilibrium constant $\left(K_{\text {eq }}\right)$ of $2.09 \pm 0.27 \times 10^{-4} \mathrm{M}$ was detected, corresponding to a redox potential ( $\mathrm{E}_{\mathrm{o}}$ ) of $-131 \mathrm{mV}$ (Fig. 4). Thiol-disulfide oxidoreductases of the thioredoxin family have redox potentials ranging from $-270 \mathrm{mV}$ for the E. coli thioredoxin to $-122 \mathrm{mV}$ for the most oxidative of all, the E. coli DsbA.

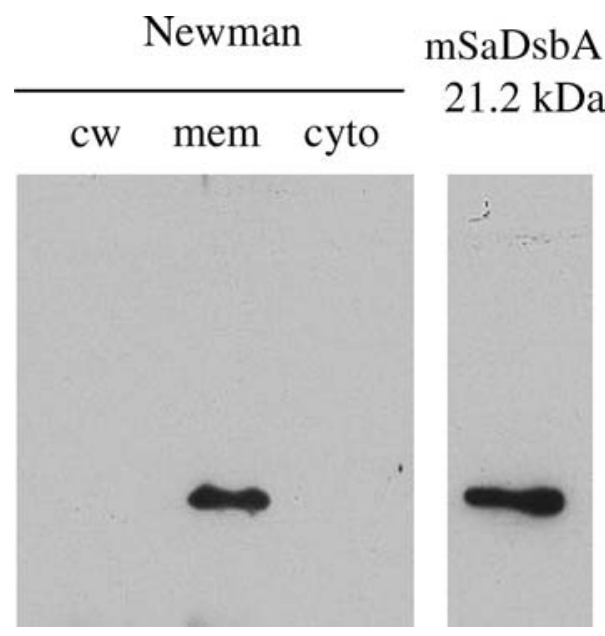

Fig. 5 Subcellular localisation of SaDsbA. Cells of strain Newman were fractionated in cell wall $(\mathrm{cw})$, cell membrane (mem) and cytoplasmic (cyto) fractions. As control, $30 \mathrm{ng}$ of purified mature staphylococcal DsbA $(m S a D s b A)$ was loaded. The protein fractions were separated on a $12 \%$ SDS-PA gel and analysed by Western blotting using the anti-DsbA antibody. The amount of protein of each fraction corresponded to a 1-ml culture

\section{Localisation of SaDsbA}

To confirm that SaDsbA is a lipoprotein, cells of strain Newman were fractionated into cell wall, cell membrane and cytoplasm. The fractions were subjected to Western blot analysis using polyclonal antibodies raised against the purified SaDsbA. An antigen corresponding to the expected size of $21.0 \mathrm{kDa}$ was detected exclusively in the membrane fraction (Fig. 5). Additionally, no SaDsbA was detected among TCA precipitated proteins obtained from the culture supernatant. In order to confirm the lipoprotein character of SaDsbA, we challenged exponentially growing cells of strain Newman with globomycin, an antibiotic which inhibits the cleavage of the lipoprotein signal sequence by blocking signal peptidase II, resulting in the accumulation of an unprocessed form of the protein that typically runs $2-3 \mathrm{kDa}$ higher than the mature lipoprotein (Inukai et al. 1984). This specific effect of globomycin has been previously used for analysis of lipoprotein maturation in $S$. aureus (Navarre et al. 1996). Protein extracts from cells treated with globomycin contained a second band running at a slightly higher molecular weight, as expected for the unprocessed SaDsbA (Fig. 6).

\section{Complementation of an $E$ coli $d s b A$ mutant}

E. coli $d s b A$ mutants are impaired in motility due to their inability to form the disulfide-bond of the flagellar protein FlgI (Jones et al. 1990; Dailey and Berg 1993). This phenotype has been used to identify genes 


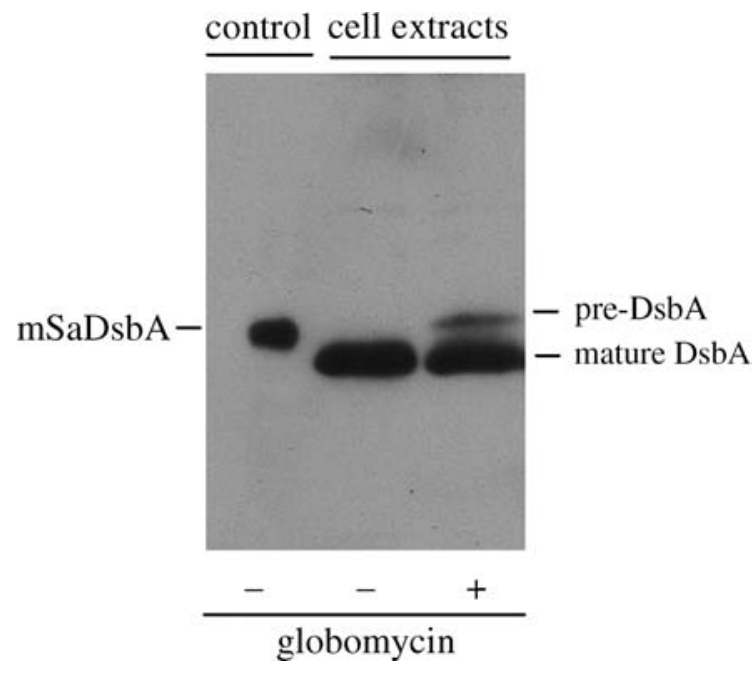

Fig. 6 Influence of globomycin on the maturation of DsbA. Bacterial cultures were treated with 0 or $50 \mu \mathrm{g} / \mathrm{ml}$ globomycin, respectively. Fifteen micrograms of proteins from whole-cell extracts were analysed by Western blotting using the anti-DsbA antibody. As control, $30 \mathrm{ng}$ of the purified (mSaDsbA) was loaded

that complement $d s b A$ mutants (Ishihara et al. 1995; $\mathrm{Ng}$ et al. 1997). To demonstrate the functionality of SaDsbA, we complemented the E. coli $d s b A$ mutant strain JCB571 with plasmid pAD2, containing SA2198 $-d s b A$. This construct clearly restored the motility of the $d s b A$ mutant, although not to wild-type levels, whereas complementation with the empty plasmid pBR322, had no effect on motility of the mutant (Fig. 7). To exclude that the efficiency of complementation was impaired because of incompatibilities of the staphylococcal gene at the transcriptional or export level in E. coli, we complemented JCB571 with plasmid pAD11, harbouring a sequence encoding a chimeric protein where the signal sequence of the $E$. coli outer membrane protein OmpA was fused to the predicted mature part of SaDsbA. Complementation of the $d s b A$ mutant with pAD11 restored the motility of JBC571 in a way that was almost identical to that obtained with pAD2 (Fig. 7). In order to assess whether the activity of SaDsbA in the E. coli mutant was dependent on the presence of $E$. coli DsbB, the motility of an $E$. coli $d s b A d s b B$ double mutant was tested in a similar way. Since it is known that cystine, a di-amino acid resulting from the oxidation of cysteine, can functionally complement a $d s b B$ mutant (Dailey and Berg 1993), the motility tests were performed on an M9-based swarm agar devoid of cysteine. On this medium, none of the plasmids encoding the $S$. aureus DsbA were able to complement either the $d s b A d s b B$ double mutant (Fig. 8), or the $d s b A$ single mutant (not shown). When the swarm agar was supplemented with $25 \mu \mathrm{M}$ cystine, the strains containing pAD2 or pAD11 partially restored motility of both the single and the double mutant (Fig. 8).

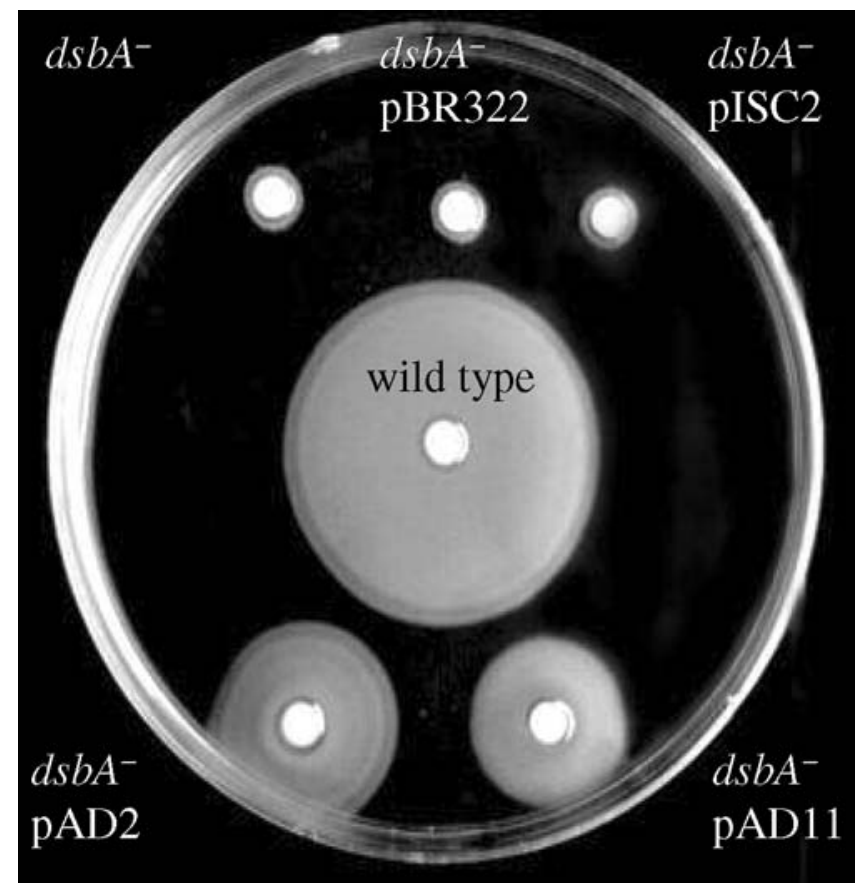

Fig. 7 Complementation of the motility of an E. coli $d s b A$ mutant. Partial restoration of the E. coli $d s b A$ deficient strain JCB571 by the staphylococcal DsbA, alone (pAD11) and in combination with SA2198 (pAD2)

Inactivation of $d s b A$ in $S$. aureus

In order to analyse the function of $d s b A$ in $S$. aureus, a $d s b A$ mutant was constructed by replacing an internal 249-bp $d s b A$ fragment with a tet $L$ resistance cassette. The $d s b A$ deficient strains were not impaired in aerobic growth. Since $d s b A$ mutants of $E$. coli have been shown to have an increased sensitivity to reduced dithiothreitol (DTT) (Missiakas et al. 1993), we tested the S. aureus mutant strains for growth inhibition by this substance. The sensitivities of strains AD21, AD22 and AD23 to this substance were the same as those of the respective wild types (data not shown). Another reported phenotype of $E$. coli $d s b A$ is a reduced resistance to the $\beta$-lactam antibiotic benzylpenicillin (Missiakas et al. 1993). Since many proteins of $S$. aureus involved in resistance to antibiotics contain cysteine residues that might form disulfide bonds, we tested the resistance of the $S$. aureus mutants to various antibiotics using $E$ test or antibiotic gradient plates. The tested antibiotics belonged to various classes, targeting cell wall biosynthesis (beta-lactams and glycopeptides), protein synthesis (aminoglycosides, lincosamides, macrolides), DNA gyrase activity (fluorochinolones) or RNA synthesis (rifampicin). However, no significant difference could be seen between wild-type and $d s b A$ mutant strains with respect to the resistance levels of all antibiotics analysed (data not shown).

The $S$. aureus beta-haemolysin, also termed sphingomyelinase $\mathrm{C}$, requires a disulfide bond for activity 


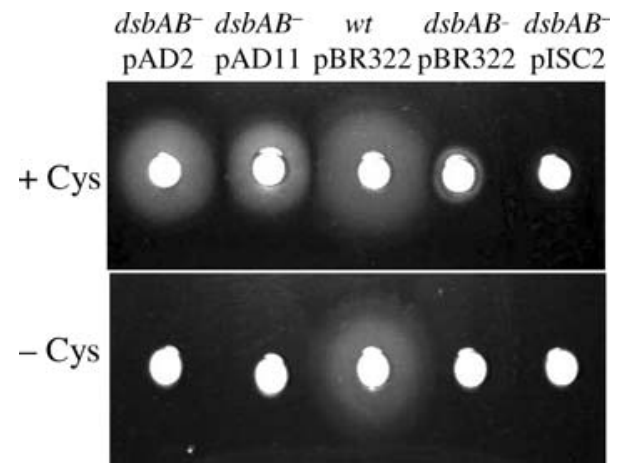

Fig. 8 Complementation of the motility of an E. coli $d s b A d s b B$ double mutant. Where indicated $(+C y s)$, cystine was added at a concentration of $25 \mu \mathrm{g} / \mathrm{ml}$

(Dziewanowska et al. 1996). We therefore compared the appearances and diameters of the beta-haemolysis-zones of the $d s b A$ defective strains AD19, AD21, AD22 and AD23 and their respective parental strains RN4220, BB270, Newman and MSSA1112 on sheep blood agar plates, but were unable to observe notable differences. Similarly, no significant differences were found in the release of haemoglobin from sheep blood cells challenged with supernatants from stationary phase cultures of the beta-haemolysin producing strain $8325-4$ or its corresponding $d s b A$ mutant AD188 (data not shown).

To assess the impact of DsbA on virulence, strain Newman and its $d s b A$ defective derivative AD22 were tested in a Caenorhabditis elegans killing assay (Sifri et al. 2003). There was no significant difference between wild type and mutant strain, neither with respect to killing rate, nor killing kinetics (C.D. Sifri, unpublished results). The same strains were also tested in a mouse model for septic arthritis (Bremell et al. 1991). The severity of arthritis, weight decrease and mortality were monitored over a period of 13 days, but no notable difference was observed between Newman and AD22 (I.M. Jonsson, unpublished results).

\section{Discussion}

The family of DsbA proteins is known to constitute the primary catalyst of protein disulfide bond formation in Gram-negative bacteria (reviewed in Kadokura et al. 2003). Members of the DsbA family have been associated in Gram-negatives with pathogenicity and penicillin resistance (Missiakas et al. 1993; Yu and Kroll 1999). With the exception of the Bdb proteins of the genus Bacillus (Ishihara et al. 1995; Bolhuis et al. 1999; Erlendsson and Hederstedt 2002), nothing is known about how disulfide bond formation is accomplished by Grampositives. We have identified a potential DsbA homologue in the human pathogen $S$. aureus. This is of particular interest, since the active forms of various staphylococcal virulence factors, such as enterotoxins and beta-haemolysin, require disulfide bond formation
(Hovde et al. 1994; Dziewanowska et al. 1996). The localisation of SaDsbA as a membrane-anchored lipoprotein supports a putative role in disulfide bond formation of excreted virulence factors.

Heterologous complementation of an E. coli $d s b A$ mutant demonstrated that SaDsbA had in fact a functional thiol-disulfide oxidoreductase activity. Moreover, the measured redox potential of SaDsbA was very similar to that of $E$. coli DsbA, indicating that the purified staphylococcal protein is strongly oxidative. However, the apparent lack of a phenotype, either in vitro or in animal infection models, leaves the role of SaDsbA in $S$. aureus open.

Despite its predominant transcription during exponential growth, Western blot analysis demonstrated the presence of SaDsbA throughout the growth. This indicated that SaDsbA showed a rather long half-life and that it might interact with surface associated, as well as excreted proteins, which are predominantly produced by $S$. aureus during the exponential and stationary growth phases, respectively. The anchoring of SaDsbA to the membrane is unusual for the oxidative members of the DsbA family, which are generally secreted through the Sec pathway and cleaved by signal peptidase I, resulting in soluble periplasmic proteins (Schierle et al. 2003). However, recently Tinsley and co-workers reported that two of the three DsbA homologues produced by the Gram-negative pathogen Neisseria meningitidis are associated with the inner membrane via a lipoprotein anchor (Tinsley et al. 2004). The particularity of neisseria to secrete toxin-filled outer-membrane vesicles (Pettit and Judd 1992) was postulated to lead to the loss of periplasmic enzymes, in particular the non-membrane-associated DsbA homologue (Tinsley et al. 2004). Gram-positive bacteria, missing an outer-membrane, have to prevent the diffusion of critical enzymes. A thick, almost impermeable cell wall defining a pseudoperiplasm, as in B. subtilis, could compensate for the lack of it (Merchante et al. 1995). B. brevis, whose DsbA homologue $\mathrm{Bdb}$ is predicted to have a cleavable signal sequence (Ishihara et al. 1995), probably makes use of this diffusion barrier. An alternative strategy to prevent diffusion of secreted proteins is anchoring them to the membrane via a lipoprotein anchor or a transmembrane anchor, as postulated for BdbD of B. subtilis (Erlendsson and Hederstedt 2002). Lipoproteins of Gram-positive bacteria have been proposed to constitute the functional equivalent of Gram-negative periplasmic proteins (Nielsen and Lampen 1982), which may be the case for SaDsbA.

In E. coli, re-oxidation of DsbA is achieved by the action of DsbB (Guilhot et al. 1995; Kishigami et al. 1995; Kishigami and Ito 1996), and in B. subtilis, BdbD is re-oxidised by $\mathrm{BdbC}$ (Erlendsson and Hederstedt 2002).

The experimental results of the present study strongly suggest that the E. coli DsbB was not able to re-oxidise SaDsbA. However, further biochemical experiments would be required to definitely confirm the lack of 
interaction between those two proteins. The absence of any DsbB or BdbC homologue, however, indicates that the re-oxidation of the staphylococcus DsbA may be carried out differently. A BLAST search through all sequenced bacterial genomes revealed that a large majority of species encoding a $\mathrm{DsbA} / \mathrm{BdbD}$ homologue also harbour a DsbB/BdbC homologue. Notable exceptions besides $S$. aureus and $S$. epidermidis were the insect symbionts Buchnera aphidicola and Wigglesworthia glossinidia. Both organisms feature very small genomes (616 respectively $697 \mathrm{~kb}$ ) that resulted from reductive genome evolution as a consequence of their endosymbiotic life forms (Akman et al. 2002; van Ham et al. 2003). Neither function nor activity of DsbA in these organisms are established, but the fact that the gene has been conserved while hundreds of others were discarded by the evolution speaks for its importance. Additionally, it suggests that some bacteria may use a different way to re-oxidise DsbA. The di-amino acid cystine for instance was shown to relieve the phenotype of an $E$. coli mutant lacking $d s b B$ at concentrations as low as $20.8 \mu \mathrm{M}$ (Dailey and Berg 1993). Interestingly, cystine is present at a concentration of $40 \mu \mathrm{M}$ in human plasma (Chawla et al. 1984; Jones et al. 2000). Since $S$. aureus is a human pathogen and is therefore often in contact with blood plasma, cystine might contribute to the re-oxidation of SaDsbA under such conditions. Another potential redox partner of SaDsbA could be SA2198. Since no homologue of SA2198 has been found outside the Staphylococcus genus, it is currently not feasible to estimate its function. The findings that $d s b A$ and SA2198 are co-transcribed, the similar genetic organisation in S. epidermidis, and that SA2198 is predicted to be localised in the same cellular compartment as SaDsbA, support the possibility that both proteins are functionally linked. However, the lack of cysteine residues in SA2198, which are essential for DsbB activity (Guilhot et al. 1995), and the observation that plasmid pAD2, containing SA2198 - $d s b A$, was not more efficient at restoring the motility of the $E$. coli mutants than plasmid pAD11, encoding only SaDsbA, seemed to exclude a DsbB-like action of SA2198 on SaDsbA.

In conclusion, despite its low sequence similarity to $E$. coli DsbA, and being a membrane anchored protein, the staphylococcal DsbA can functionally replace $E$. coli DsbA. SaDsbA is thus the first $S$. aureus protein shown to have the potential for extracellular disulfide bond formation. The finding that $S$. aureus, as well as some other bacteria, encode a DsbA but lack a DsbB homologue, leads us to postulate the existence of an alternative disulfide formation pathway distinct from the classical DsbA-DsbB electron cascade. The exact function of SaDsbA in staphylococci, and its importance for virulence of the human pathogens $S$. aureus and $S$. epidermidis remains to be elucidated.

Acknowledgments We thank J.C.A. Bardwell for providing the strain JCB570 and JCB571, I.M. Jonsson and C. Sifri for performing the animal studies and Sankyo Corp (Tokyo, Japan) for the kind gift of globomycin. This work was supported by a grant from the Olga Mayenfisch Foundation, and the SNF grants 31105390 and $3100 \mathrm{~A} 0-100234$.

\section{References}

Akman L, Yamashita A, Watanabe H, Oshima K, Shiba T, Hattori M, Aksoy S, Yamashita A, Watanabe H, Oshima K, Shiba T, Hattori M, Aksoy S (2002) Genome sequence of the endocellular obligate symbiont of tsetse flies, Wigglesworthia glossinidia. Nat Genet 32:402-407

Altschul S, Gish W, Miller W, Myers E, Lipman D (1990) Basic local alignment search tool. J Mol Biol 215:403-410

Anfinsen CB (1973) Principles that govern the folding of protein chains. Science 181:223-230

Bader M, Muse W, Zander T, Bardwell J (1998) Reconstitution of a protein disulfide catalytic system. J Biol Chem 273:10302-10307

Bader M, Winther JR, Bardwell JC (1999) Protein oxidation: prime suspect found 'not guilty'. Nat Cell Biol 1:E57-E58

Bardwell J, McGovern K, Beckwith J (1991) Identification of a protein required for disulfide bond formation in vivo. Cell 67:581-589

Bendtsen JD, Nielsen H, von Heijne G, Brunak S (2004) Improved prediction of signal peptides: SignalP 3.0. J Mol Biol 340:783795

Berger-Bächi B (1983) Increase in transduction efficiency of Tn 551 mediated by the methicillin resistance marker. J Bacteriol 154:533-535

Berger-Bächi B, Kohler ML (1983) A novel site on the chromosome of Staphylococcus aureus influencing the level of methicillin resistance: genetic mapping. FEMS Microbiol Lett 20:305-309

Bischoff M, Entenza JM, Giachino P (2001) Influence of a functional $\operatorname{sig} B$ operon on the global regulators $\operatorname{sar}$ and $a g r$ in Staphylococcus aureus. J Bacteriol 183:5171-5179

Bolhuis A, Venema G, Quax W, Bron S, van Dijl J (1999) Functional analysis of paralogous thiol-disulfide oxidoreductases in Bacillus subtilis. J Biol Chem 274:24531-24538

Bolivar F, Rodriguez R, Greene P, Betlach MC, Heyneker HL, Boyer HW (1977) Construction and characterisation of new cloning vehicles II A multipurpose cloning system. Gene 2:95113

Bremell T, Lange S, Yacoub A, Ryden C, Tarkowski A (1991) Experimental Staphylococcus aureus arthritis in mice. Infect Immun 59:2615-2623

Brückner R (1997) Gene replacement in Staphylococcus carnosus and Staphylococcus xylosus. FEMS Microbiol Lett 151:1-8

Burall LS, Harro JM, Li X, Lockatell CV, Himpsl SD, Hebel JR, Johnson DE, Mobley HL (2004) Proteus mirabilis genes that contribute to pathogenesis of urinary tract infection: identification of 25 signature-tagged mutants attenuated at least 100fold. Infect Immun 72:2922-2938

Charbonnier J, Belin P, Moutiez M, Stura EA, Quéméneur E (1999) On the role of the cis-proline residue in the active site of DsbA. Protein Sci 8:96-105

Chawla RK, Lewis FW, Kutner MH, Bate DM, Roy RG, Rudman D (1984) Plasma cysteine, cystine, and glutathione in cirrhosis. Gastroenterology 87:770-776

Cheung AL, Eberhardt KJ, Fischetti VA (1994) A method to isolate RNA from Gram-positive bacteria and mycobacteria. Anal Biochem 222:511-514

Dailey F, Berg H (1993) Mutants in disulfide bond formation that disrupt flagellar assembly in Escherichia coli. Proc Natl Acad Sci USA 90:1043-1047

Duthie E, Lorenz L (1952) Staphylococcal coagulase: mode of action and antigenicity. J Gen Microbiol 6:95-107

Dziewanowska K, Edwards V, Deringer J, Bohach G, Guerra D (1996) Comparison of the $\beta$-toxins from Staphylococcus aureus and Staphylococcus intermedius. Arch Biochem Biophys 335:102-108 
Erlendsson L, Hederstedt L (2002) Mutations in the thiol-disulfide oxidoreductases $\mathrm{BdbC}$ and $\mathrm{BdbD}$ can suppress cytochrome $c$ deficiency of CcdA-defective Bacillus subtilis cells. J Bacteriol 184:1423-1429

Fabianek R, Hennecke H, Thöny-Meyer L (1998) The active-site cysteines of the periplasmic thioredoxin-like CcmG of Escherichia coli are important but not essential for cytochrome $c$ maturation in vivo. J Bacteriol 180:1947-1950

Fabianek R, Hennecke H, Thöny-Meyer L (2000) Periplasmic protein thiol:disulphide oxidoreductases of Escherischia coli. FEMS Microbiol Lett 24:303-316

Giachino P, Engelmann S, Bischoff M (2001) $\sigma^{\mathrm{B}}$ activity depends on RsbU in Staphylococcus aureus. J Bacteriol 183:1843-1852

Grauschopf U, Fritz A, Glockshuber R (2003) Mechanism of the electron transfer catalyst DsbB from Escherichia coli. EMBO J 22:3503-3513

Guilhot C, Jander G, Martin N, Beckwith J (1995) Evidence that the pathway of disulfide bond formation in Escherichia coli involves interactions between the cysteines of DsbA and DsbB. Proc Natl Acad Sci USA 92:9895-9899

Ha UH, Wang Y, Jin S (2003) DsbA of Pseudomonas aeruginosa is essential for multiple virulence factors. Infect Immun 71:15901595

van Ham RCHJ, Kamerbeek J, Palacios C, Rausell C, Abascal F, Bastolla U, Fernandez JM, Jimenez L, Postigo M, Silva FJ, Tamames J, Viguera E, Latorre A, Valencia A, Moran F, Moya A (2003) Reductive genome evolution in Buchnera aphidicola. Proc Natl Acad Sci USA 100:581-586

Helmann JD (1995) Compilation and analysis of Bacillus subtilis $\sigma^{\mathrm{A}}$-dependent promoter sequences: evidence for extended contact between RNA polymerase and upstream promoter DNA. Nucleic Acid Res 23:2351-2360

Hovde CJ, Marr JC, Hoffmann ML, Hackett SP, Chi Y, Crum KK, Stevens DL, Stauffacher CV, Bohach GA (1994) Investigation of the role of the disulphide bond in the activity and structure of staphylococcal enterotoxin C1. Mol Microbiol 13:897-909

Huber-Wunderlich M, Glockshuber R (1998) A single dipeptide sequence modulates the redox properties of a whole enzyme family. Fold Des 3:161-171

Inukai M, Ghrayeb J, Nakamura K, Inouye M (1984) Apolipoprotein, an intermediate in the processing of the major lipoprotein of the Escherichia coli outer membrane. J Biol Chem 259:757-760

Ishihara T, Hideaki T, Hasegawa Y, Tsukagoshi N, Yamagata H, Udaka S (1995) Cloning and characterization of the gene for a protein:thiol-disulfide oxidoreductase in Bacillus brevis. J Bacteriol 177:745-749

Jonda S, Huber-Wunderlich M, Glockshuber R, Mossner E (1999) Complementation of DsbA deficiency with secreted thioredoxin variants reveals the crucial role of an efficient dithiol oxidant for catalyzed protein folding in the bacterial periplasm. Embo $\mathbf{J}$ 18:3271-3281

Jones C, Macnab R, Okino H, Aizawa S (1990) Stoichiometric analysis of the flagellar hook- (basal-body) complex of Salmonella typhimurium. J Mol Biol 212:377-387

Jones D, Carlson J, Mody V, Cai J, Lynn M, Sternberg P (2000) Redox state of glutathione in human plasma. Free Radic Biol Med 28:625-635

Kadokura H, Katzen F, Beckwith J (2003) Protein disulfide bond formation in prokaryotes. Annu Rev Biochem 72:111-135

Kadokura H, Tian H, Zander T, Bardwell J, Beckwith J (2004) Snapshots of DsbA in action: detection of proteins in the process of oxidative folding. Science 303:534-537

Kishigami S, Ito K (1996) Roles of cysteine residues of DsbB in its activity to reoxidize DsbA, the protein disulphide bond catalyst of Escherichia coli. Genes Cells 1:201-208

Kishigami S, Kanaya E, Kikuchi M, Ito K (1995) DsbA-DsbB interaction through their active site cysteines. J Biol Chem 270:17072-17074
Kuroda M, Ohta T, Uchiyama I, Baba T, Yuzawa H, Kobayashi I, Cui L, Oguchi A, Aoki K, Nagai Y, Lian J, Ito T, Kanamori M, Matsumaru H, Maruyama A, Murakami H, Hosoyama A, Mizutani-Ui Y, Kobayashi N, Sawano T, Inoue R, Kaito C, Sekimizu K, Hirakawa H, Kuhara S, Goto S, Yabuzaki J, Kanehisa M, Yamashita A, Oshima K, Furuya K, Yoshino C, Shiba T, Hattori M, Ogasawara N, Hayashi H, Hiramatsu K (2001) Whole genome sequencing of meticillin-resistant Staphylococcus aureus. Lancet 357:1225-1240

Martin JL (1995) Thioredoxin-a fold for all reasons. Structure $3: 245-250$

Meima R, Eschevins C, Fillinger S, Bolhuis A, Hamoen LW, Dorenbos R, Quax WJ, van Dijl JM, Provvedi R, Chen I, Dubnau D, Bron S (2002) The bdbDC operon of Bacillus subtilis encodes thiol-disulfide oxidoreductases required for competence development. J Biol Chem 277:6994-7001

Merchante R, Pooley H, Karamata D (1995) A periplasm in Bacillus subtilis. J Bacteriol 177:6176-6183

Miki T, Okada N, Danbara H (2004) Two periplasmic disulfide oxidoreductases, DsbA and SrgA, target outer membrane protein SpiA, a component of the Salmonella pathogenicity island 2 type III secretion system. J Biol Chem 279:3463134642

Missiakas D, Georgopoulos C, Raina S (1993) Identification and characterization of the Escherichia coli gene $d s b B$, whose product is involved in the formation of disulfide bonds in vivo. Proc Natl Acad Sci USA 90:7084-7088

Navarre WW, Daefler S, Schneewind O (1996) Cell wall sorting of lipoproteins in Staphylococcus aureus. J Bacteriol 178:441-446

$\mathrm{Ng}$ T, Kwik J, Maier R (1997) Cloning and expression of the gene for a protein disulfide oxidoreductase from Azotobacter vinelandii: complementation of an Escherichia coli $d s b A$ mutant strain. Gene 188:109-113

Nielsen J, Lampen J (1982) Glyceride-cysteine lipoproteins and secretion by Gram-positive bacteria. J Bacteriol 152:315-322

Nilsson IM, Lee JC, Bremell T, Ryden C, Tarkowski A (1997) The role of staphylococcal polysaccharide microcapsule expression in septicemia and septic arthritis. Infect Immun 65:4216-4221

Peng HL, Novick RP, Kreiswirth B, Kornblum J, Schlievert P (1988) Cloning, characterization, and sequencing of an accessory gene regulator (agr) in Staphylococcus aureus. J Bacteriol 170:4365-4372

Pettit RK, Judd RC (1992) The interaction of naturally elaborated blebs from serum-susceptible and serum-resistant strains of Neisseria gonorrhoeae with normal human serum. Mol Microbiol 6:729-734

Rao L, Karls RK, Betley MJ (1995) In vitro transcription of pathogenesis-related genes by purified RNA polymerase from Staphylococcus aureus. J Bacteriol 177:2609-2614

Rhoads Kraemer G, Iandolo J (1990) High-frequency transformation of Staphylococcus aureus by electroporation. Curr Microbiol 21:373-376

Rossi J, Bischoff M, Wada A, Berger-Bächi B (2003) MsrR, a putative cell envelope-associated element involved in Staphylococcus aureus sarA attenuation. Antimicrob Agents Chemother 47:2558-2564

Sambrook J, Fritsch EF, Maniatis T (eds) (1989) Molecular cloning, a laboratory manual, 2nd edn. Cold Spring Harbor Laboratory, New York

Schierle CF, Berkmen M, Huber D, Kumamoto C, Boyd D, Beckwith J (2003) The DsbA signal sequence directs efficient, cotranslational export of passenger proteins to the Escherichia coli periplasm via the signal recognition particle pathway. J Bacteriol 185:5706-5713

Schneewind O, Mihaylova-Petkov D, Model P (1993) Cell wall sorting signals in surface proteins of Gram-positive bacteria. EMBO J 12:4803-4811

Sifri CD, Begun J, Ausubel FM, Calderwood SB (2003) Caenorhabditis elegans as a model host for Staphylococcus aureus pathogenesis. Infect Immun 71:2208-2217 
Stenson TH, Weiss AA (2002) DsbA and DsbC are required for secretion of pertussis toxin by Bordetella pertussis. Infect Immun 70:2297-2303

Sutcliffe IC, Harrington DJ (2002) Pattern searches for the identification of putative lipoprotein genes in Gram-positive bacterial genomes. Microbiology 148:2065-2077

Thompson J, Higgins D, Gibson T (1994) CLUSTAL W: improving the sensitivity of progressive multiple sequence alignment through sequence weighting, position-specific gap penalties and weight matrix choice. Nucleic Acids Res 22:46734680

Thöny-Meyer L, Künzler P, Hennecke H (1998) Requirements for maturation of Bradyrhizobium japonicum cytochrome c550 in Escherichia coli. Eur J Biochem 235:754-761

Tinsley CR, Voulhoux R, Beretti J-L, Tommassen J, Nassif X (2004) Three homologues, including two membrane-bound proteins, of the disulfide oxidoreductase DsbA in Neisseria meningitidis: effects on bacterial growth and biogenesis of functional type IV pili. J Biol Chem 279:27078-27087

Tjalsma H, Bolhuis A, Jongbloed J, Bron S, van Dijl J (2000) Signal peptide-dependent protein transport in Bacillus subtilis: a genome-based survey of the secretome. Microbiol Mol Biol Rey 64:515-547

Wada A (2001) An improved method for purifying bacterial genomic DNAs for direct sequencing by capillary automated sequencer. TTO 1:T02049

Yu J, Kroll JS (1999) DsbA: a protein-folding catalyst contributing to bacterial virulence. Microbes Infect 1:1221-1228

Yu J, Oragui EE, Stephens A, Kroll JS, Venkatesan MM (2001) Inactivation of DsbA alters the behaviour of Shigella flexneri towards murine and human-derived macrophage-like cells. FEMS Microbiol Lett 204:81-88 